\title{
Attachment Styles Are Related to ERPs Elicited to Angry Faces in an Oddball Paradigm
}

\author{
Ruth E. Mark ${ }^{1 *}$, Fienke I. M. Geurdes ${ }^{1}$, Marrie H. J. Bekker ${ }^{2}$ \\ ${ }^{1}$ Department of Medical Psychology and Neuropsychology, Tilburg University, Tilburg, The Netherlands \\ ${ }^{2}$ Department of Clinical Psychology, Tilburg University, Tilburg, The Netherlands \\ Email: *R.E.Mark@uvt.nl
}

Received May 19, 2011; revised July 15, 2011; accepted October 24, 2011

\begin{abstract}
Attachment theory suggests that anxious attachment is associated with hypervigilance to threatening social stimuli, and avoidant attachment with avoidance or suppression of processing such stimuli. Twenty-five students viewed angry, fearful and neutral female faces in four visual oddball tasks, and completed the Attachment Style Questionnaire, the Autonomy-Connectedness Scale, and Anxiety and Depression subscales of the Symptom Checklist-90. When the oddballs were angry faces in a background of neutral frequents, we found higher levels of autonomy and secure attachment to be related to larger N100 and smaller P300 amplitudes; higher levels of anxious attachment were, on the contrary, associated with smaller N100 and larger P300 amplitudes. Variation in attachment is related to approaching, or withdrawing from threatening stimuli, and ERP-techniques add to our understanding of how the attachment system actually works.
\end{abstract}

Keywords: Event-Related Potentials; Attachment Styles or Dimensions; Autonomy; Anxiety; Attentional Bias Theory

\section{Introduction}

Neuropsychologists have long since claimed that early experiences mold the brain of the adult that the child will become. Few studies have directly examined whether early childhood experiences do change the way the brain develops or how these affect the way pertinent stimuli are processed in adulthood. Longitudinal studies from childhood to adulthood are undoubtedly the best way to tackle this problem. Unfortunately these kinds of studies tend to be time, labor and financially prohibitive. Retrospective and/or cross-sectional studies also have their difficulties not least when trying to control for confounding variables, many of which can have profound effects on neurological development. Such variable control may actually be impossible even should the experimenter decide to investigate on a case-by-case basis. Attachment theory and Event Related Potential methodology may be a way forward when attempting to unravel the conundrum of how early childhood experiences may influence brain development and ultimately how individuals with different attachment styles process the stimuli in the world around them.

Attachment theory was first introduced by Bowlby in the late 1960s [1-4] and since then there have been an abundance of studies in this field. The theory suggests that children internalize early experiences with the pri-

\footnotetext{
${ }^{*}$ Corresponding author.
}

mary caregiver (typically the mother) and in this way form the basis for all their future relationships. These mental representations or maps which Bowlby called "internal working models" are very similar to Beck's "cognitive schemata" [5]. They are believed to be laid down early in childhood and represent the self and others and the (attachment) relationships between them. That these schemata persist into adulthood is also widely accepted in the literature as is the notion that they determine not only our relationships with our parents but also our romantic liaisons and more generally how we get along with other people [6]. While they are likely to be modified as we experience a variety of (romantic) relationships, a key assumption in attachment theory is that the basic schemata are formed in childhood and that they do not fundamentally change as we age. In this sense they are stable and "trait-like".

There has been some recent criticism of the trait approach in that 1) researchers have found that people may have different attachment patterns across different relationships and 2) the test-retest stability of attachment styles is low [7,8]. While Fraley [9] suggested that a connectionist model (where the context of the relationship is crucial) may be more appropriate than a trait model when discussing adult attachment most researchers maintain a trait approach convinced that the relationship that develops between the infant and the primary attachment person is crucial for all of the child's future relationships. 
There is also some debate in the literature with respect to how many attachment styles exist (ranging from two to at least five, see e.g. [10]). Ainsworth [11] developed the Strange Situation test and identified three distinct patterns of infant attachment: secure, anxious-resistant, and avoidant. Securely attached children were happy to see their carer (typically their mother) after a separation and if they were upset they were easily comforted. Anxious-resistant children were ambivalent towards their carer on that person's return and could not be comforted. Avoidant children rejected their carer on reunion and avoided contact with him/her. Other researchers talk about a dimensional approach where they recognise that it is possible to be both anxious and avoidant or neither (the latter is then referred to as "secure"). Insecure attachment styles have been linked with many psychological disorders including: depression, anxiety, eating disorders, antisocial behavior and more [12-16]. It is however certainly not the case that everyone who has an insecure attachment style also has some sort of psychopathological disorder while at the same time it appears to be the case that some psychopathologies are rooted in insecure attachment in childhood [17]. Indeed, attachment has been referred to as a "relational emotion regulation system" (e.g. [18] p. 446), while insecure attachment has been associated with the development of anxiety disorders (see [19] for a review). As has already been suggested, these attachment styles persist into adulthood and are believed to have important influences on how individuals attend to and memorize (especially personallyrelevant and threatening) information [20]. Bowlby [1,2] labelled secure attachment as autonomy, a healthy psychological condition typically reached by the end of adolescence. Problems with autonomy have also been associated (like insecure attachment) with anxiety and depression [21]. As autonomy components contribute to anxiety independently to anxious attachment [22] we also focused on autonomy in the present study.

How the attachment system actually works has been a matter for debate. Bowlby's seminal work suggested that threatening social stimuli were very likely to activate the attachment system and Main [23] stated that attentional factors were crucial in the regulation of this system. Further, attachment theory suggests that anxiously attached individuals should approach threatening social stimuli/ events or at least show a vigilance for detecting them in the environment, while avoidant individuals should avoid threat. Brennan et al. [24] suggested that in anxiouslyattached individuals the attachment system operated in a hyperactive mode while in those with avoidant attachment the system operated in deactivation mode. Unfortunately the literature does not always support these basic assumptions. Main et al. [20] found for example avoidance of attachment-related pictures in both anxious and avoidant individuals and Dewitte et al. [25] using a dotprobe paradigm found that both anxious and avoidant individuals avoided attachment-related threatening word stimuli.

Whether individuals approach or avoid social threat seems to depend largely on the context and on the length of stimulus exposure time. It may be the case that anxiously attached individuals initially approach social threat but quickly push it mentally away from themselves (i.e. avoid processing it further). This is backed up by the extensive literature on attentional biases to all sorts of threatening stimuli in anxious individuals where, depending on the task used, highly anxious individuals in the normal (and clinical) population show attentional vigilance to threat (so-called attentional bias to, especially personally-relevant, threat) and then subsequently avoid it. This has been referred to as the Double movement/ Hypervigilance-avoidance theory [26]. Whether this is also the case in anxiously-attached individuals or not is largely not known although Mikulincer et al. [27] did find more vigilance to emotional, attachment-related information in their anxiously-attached subjects. Whether avoidant individuals avoid threatening stimuli from the moment they perceive them is also largely unknown. Despite these uncertainties, some researchers [28-30] have suggested that there is a great deal of similarity between attachment theory and the attentional bias theory of anxiety.

The main problem in both attachment and attentionalbias studies is that the timing of stimulus processing is difficult to monitor. Most studies have used for example emotional Stroop tests or other reaction-time tasks where timing of stimulus processing before the motor response has been made is not possible. Event-related potentials (ERPs) can productively be used to assess whether people with different attachment styles approach and/or avoid threatening stimuli. Holmes [31] suggested that attachment theory could perhaps provide "a bridge between the biological and the psychological with important implications for psychotherapeutic theory and practice" (p. 430).

Since his remarks however, and despite the burgeoning field of social cognitive neuroscience where there is an "increasing recognition that social forces have shaped brain evolution" ([32], p. 254) few studies have directly examined individuals with different attachment styles using psychophysiological measures. ERPs have the advantage over other brain scanning methods in that they provide detailed temporal information and that they let us examine how a person processes a stimulus from its presentation until a response is required. While most studies on attachment and attentional-bias use reactiontime tasks, which focus on the final motor response, event-related potentials (ERPs) can productively be used 
to assess to what degree the two main insecure attachment styles are related to approaching and/or avoidance of threatening stimuli. We used ERP methodology in the current study to investigate more directly than behavioral studies allow whether participants approached (showed an attentional bias) or withdrew (showed a cognitive avoidance) from threatening facial expressions and if this was related to their attachment styles. Before outlining our study aims and main hypotheses in more detail we will, below, first briefly explain how ERPs may help in obtaining insight into attachment-related information processing.

ERP components have been linked with specific aspects of information processing. The early negative N100 component has been associated with attentional processes and is believed to be sensitive to physical stimulus factors [33]. The P200 is believed to reflect early stimulus discrimination while the N200 appears to be sensitive to both the arousal levels of stimuli [33] and with stimulus identification and differentiation [34]. The majority of studies have however focused on the P300 or Late Positive Potential which can be best elicited by some variant of the oddball paradigm. Many theories abound as to what the P300 measures including: context updating [35], context closure [36], attention allocation and activation of immediate memory [37], and stimulus evaluation and response selection [38].

Many studies have been carried out using the ERP to assess how individuals process emotional stimuli. In general P300 amplitudes are augmented to emotionally salient stimuli in comparison to neutral stimuli [39-41]. Larger P300 amplitudes to emotional stimuli suggest that these stimuli are processed more deeply or fully in some way [42]. Fewer significances have emerged for P300 latencies and arousal effects have been found more consistently than valence effects ([33], but see [43] for stronger effects to unpleasant versus pleasant stimuli). The source of the P300 has been traced to the amygdala-parietal cortex [44] and the amygdala (among other regions including prefrontal cortex) has of course also been implicated in the integration of emotional and cognitive processes [45]. The amygdala may however very well prove to have subnuclei with different functions. It is not currently clear if specific networks/neural systems are employed in the processing of different emotional facial expressions [30]. For example, the pons may be activated when anger [46] is the emotion to be processed while fear, happiness and disgust have all been linked with amygdala activation $[47,48]$ and sadness with the putamen [49].

Faces depicting emotional expressions have been a popular choice of stimuli in many ERP studies of emotion largely due to their evolutionary relevance. As Ochsner [32] points out "the face... conveys a wealth of socially relevant information" (p. 254), and Bar-Haim et al. [50] stated that: "An angry or fearful facial expression is a natural sign of potential threat, whereas a threat word is an arbitrary symbol" (p. 13). Angry faces are universally seen as cues for interpersonal threat while faces depicting fear are a more indirect sign of threat and depend more on the individual observing it (fearful faces can be interpreted as more threatening in the socially-anxious, see for example [51]). In Eimer and Holmes' [52] review of ERPs to emotional faces the consensus seems to be that these stimuli when compared with neutral expressions increase the amplitudes of positive ERP components and that this effect starts early at frontocentral sites at around 120 - $180 \mathrm{~ms}$ post-stimulus.

Individual differences appear to be crucial when determining how someone will process threat. Sugiura et al. [53] suggested that the neural activity of the cerebral cortex may very well be linked with specific aspects of personality and Canli et al. [54] found that the brain's reactivity to emotional stimuli could be predicted from an individual's score on the dimensions of extraversion and neuroticism. Van Assen and Bekker [55] recently found however that autonomy-connectedness, the adult psychological condition which results from secure attachment, was relatively independent from personality factors as measured by the Big Five. From an attachment theory viewpoint this makes sense in that everyone, irrespective of personality type, should ultimately be able to reach a state of secure attachment.

Few studies have directly assessed the attentional bias/ cognitive avoidance theory of general anxiety using ERP technology [56]. One recent study [57] that did used an attention-shifting paradigm. They found only one significant group interaction: P200 amplitudes were larger for anxious compared to non-anxious participants at site $\mathrm{Cz}$, but only for angry faces (not for neutral, fearful, sad or happy faces), while behaviorally anxious subjects were slower to targets regardless of the emotion expressed by the face cues. Bar-Haim et al. [50] concluded that ERPs were more sensitive than behavioral measures in picking up attentional biases in anxiety.

This attentional bias to threat in highly anxious subjects has been found in a wide variety of studies. Consensus as to what the underlying mechanisms of this bias are is lacking however. Another problem in this field is how anxiety is measured (effects tend to be stronger for state than trait measures), which populations are tested and the wide variety of stimuli and/or tasks that have been employed making comparison across studies difficult.

Few studies to date have used ERPs to assess how different attachment styles are associated with the processing of (relevant) emotional stimuli. Zhang, et al. [57] using facial expressions (happy, fearful and neutral) as stimuli in a backward masking paradigm (with two con- 
ditions: supraliminal and subliminal) divided their 30 male subjects into three attachment-style groups: (secure, anxious, avoidant) using the ECR [24]. No significant group main effects or group $\mathrm{x}$ facial expressions interacttions were found. Interactions were found with attachment-style and electrode site with avoidant showing less negativity (N200 and N400) and more positivity (P200) than anxious or secure participants. These authors suggested that attachment style differences could potentially influence any or all stages of information processing. However, the absence of significant group effects and group $\mathrm{x}$ type of facial expression interactions suggests that this interpretation must be made with caution. Perhaps using attachment style and autonomy as continuous variables instead of categories might be more promising. This is what we attempt to do in the present study.

Zilber, et al. [58] asked participants with different attachment styles (also based on the ECR) to rate the valence of unpleasant, pleasant and neutral pictures from the IAPS database [59] while measureing their ERPs. Anxiously-attached participants had larger Late Positive Potential (LPP) amplitudes to negative pictures than the other groups. They took this positive shift in the ERP to reflect hyperactivation but said that this was only active during the later stages of information processing.

In a third study, Zayas et al. [60] using a lexical decision task found larger N400 amplitudes to rejection-related words than to acceptance-related words, which was more pronounced in anxiously-attached and less pronounced for avoidantly attached individuals. N400 amplitudes in such lexical decision tasks reflect heightened neural responses to deviant/not expected words at the end of sentences [61]. Zayas et al.'s findings are difficult to interpret, but might reflect heightened surprise to rejection-versus acceptance-related words in the insecurely attached. In other words their brains might be more sensitive to negatively-toned, attachment-related stimuli.

Finally and very recently, Fraedrich, et al. [62] examined emotional face processing in 16 mothers using infant emotional faces (with positive, negative and/or neutral expressions) in a three-stimulus oddball paradigm. They found that insecure (versus secure) mothers had enhanced amplitudes for the face-sensitive N170 component and smaller N200s. Secure mothers had larger P300 amplitudes compared to the insecure group which, as the authors suggested, could reflect a perceptual bias to social stimuli in the securely attached.

\section{The Current Study: Main Aims and Hypotheses}

The main differences between the current study and those which have been conducted to date were that we used a simplified oddball task and instead of dividing participants into extreme groups we used their individual scores from the three questionnaires (see below) as con- tinuous variables. We also measured trait anxiety and autonomy in our adult female participants, something previous studies have not done. We used an oddball task because it is widely recognised as one of the best ways to obtain the ERP components we wanted to measure.

We expected high levels of insecure, anxious attachment to be initially related to approaching, and then, later in the time course of stimulus processing, to avoidance of emotionally threatening face oddballs (i.e., we expected them to respond like individuals with high trait anxiety according to the attentional bias/cognitive avoidance theory for anxiety). We hypothesized this to be reflected in heightened N100/P200 amplitudes (increased attention) and reduced P300 (reduced elaboration) amplitudes. We expected that high levels of insecure avoidant attachment would be related to suppression of processing the threatening face oddballs resulting in a flattening of the overall waveform (i.e. both reduced N100 and P300 amplitudes). We also expected that participants with both high levels of secure attachment and autonomy would show less attention to but more elaborate later processing of the threatening face oddballs, reflected in N100s smaller than those for anxiously attached subjects and larger P300s (the latter due to the fact that emotional stimuli tend to be processed more deeply by subjects, see page 3 above). We also measured trait anxiety and depression in order to assess if the ERPs were similar for subjects with high levels of anxious attachment and general anxiety. Finally, we expected to see stronger effects with angry faces (these are linked with interpersonal threat, see, e.g., [51] and when those faces were oddballs (oddballs typically produce larger P300 amplitudes than frequents, e.g. [35].

\section{Methods}

\subsection{Participants}

The sample consisted of 27 neurologically healthy female students with normal or corrected to normal vision $(M=22.4$ years, $S D=1.2$, range $=18-27)$. Most ERP studies use fewer than 16 participants. All participants signed an informed consent form before taking part in the experiment. After data assessment, two participants were excluded due to artifacts in the ERP data, leaving a total of 25 for data analysis.

\subsection{Assessment of Attachment, Autonomy and Anxiety/Depression}

Participants filled in various questionnaires immediately after they completed the experiment. Attachment was assessed using the Attachment Style Questionnaire (ASQ; [63]), a 40-item Likert-type questionnaire designed to measure five dimensions of adult attachment, namely Confidence ( 8 items), Discomfort with closeness (10 items), Need for approval (7 items), Preoccupation with 
relationships (8 items), and Relationships as secondary (7 items). Several previous studies $[16,64,65]$ have shown that these five subscales loaded on to two factors, namely anxious and avoidant attachment, while at the same time Confidence (expressing secure attachment) loaded negatively on both. We therefore reduced the number of attachment subscales to three, namely: Anxiously attached (Need for approval plus Preoccupation with relationships); Avoidantly attached (Discomfort with closeness plus Relationships as secondary); and Securely attached (Confidence). Cronbach's alphas were 0.91 for Anxiously attached, 0.81 for Avoidantly attached, and 0.72 for Securely attached.

Autonomy was assessed using the Autonomy-Connectedness scale (ACS-[51,15]). The three subscales are Selfawareness, Sensitivity to others, and Capacity for managing new situations [15] The ACS-30 is a reliable and valid measure $[15,66]$. Cronbach's alphas in the current study were $0.83,0.88$, and 0.76 for Self-awareness, Sensitivity to others, and Capacity for managing new situations, respectively.

Finally, the Symptom Checklist-90 (SCL-90; [67]) was used to assess anxiety and depression. From the 9 subscales we used Depression (16 items) and Anxiety (10 items). Each item is rated on a 5-point Likert scale, ranging from 1 (not at all) to 5 (very much so). Cronbach's alphas in the current study were 0.93 for Depression and 0.91 for Anxiety.

\subsection{Stimuli}

Two caucasian female faces with separate identities were chosen from the Matsumoto and Ekman [68] database. We chose female faces because the mother is generally considered the first most important attachment figure. For the first identity we chose one angry and one neutral emotional expression, and for the second identity one fearful and one neutral emotional expression.

Four visual, two-stimulus oddball tasks were created. The oddball stimulus occurred $30 \%$ of the time and the frequent stimulus occurred $70 \%$ of the time. In task 1 and 2 the same female identity was used with either an angry (task 1) or a neutral (task 2) expression as the oddball presented in the same session with the neutral (task 1) or angry (task 2) expression acting as the frequent stimuli. In task 3 and 4 a different female identity was used with either a fearful (task 3 ) or a neutral (task 4) expression as the oddball presented in the same session with the neutral (task 3) or fearful (task 4) expression acting as the frequent stimuli. The test items were displayed in central vision at a moderate contrast on a computer monitor. Each face was presented full-screen for $200 \mathrm{~ms}$ with an interstimulus interval (ISI) which randomly varied between $600-800 \mathrm{~ms}$ in order to avoid expectations for stimulus onset.

\subsection{Experimental Procedure}

ERP assessment took place in the experimental laboratory at Tilburg University, and lasted for about one hour. The presentation order of the four oddball tasks was counterbalanced across subjects. Following electrode application (see below), participants were seated in a dark, sound-attenuated cabin with the stimulus presentation monitor placed at a one meter viewing distance. After a short practice task the participants began the main experiment. They were instructed to press a button in their right hand as quickly and accurately as possible every time they saw a deviant (oddball) stimulus. There was a 5 minute pause between each task (when participants simply chatted with the experimenter) and each trial began with the presentation of a fixation point (a white cross in the middle of the screen) for $300 \mathrm{~ms}$ which was erased $100 \mathrm{~ms}$ prior to the presentation of each stimulus.

\subsection{Analysis of ERP Data}

Continuous EEG was recorded by means of the ActiveTwo System (BioSemi AactiveTwo, Amsterdam) from the scalp at a sampling rate of $256 \mathrm{~Hz}$ from 8 electrodes embedded in an elastic cap that were referenced to the left mastoid. The electrodes were positioned according to the 10 - 20 system [69] at four midline sites $(\mathrm{Fz}, \mathrm{Cz}, \mathrm{Pz}$, $\mathrm{Oz})$, at two over the left (F3, C3) and right (F4, C4) hemispheres, and at the left and right mastoid processes. A horizontal EOG was recorded from a pair of electrodes placed on the outer canthi of both eyes and a vertical EOG was recorded via a pair of electrodes placed on the infra-orbital and supra-orbital ridges of the left eye. Impedance for all electrodes was kept below $5 \mathrm{k} \Omega$.

The data were analysed offline using BrainVision Analyzer software (BrainProducts $\mathrm{GmbH}$ ). A $0.03-30 \mathrm{~Hz}$, $48 \mathrm{~dB} /$ octave band-pass filter was applied to the EEG signal and the data were re-referenced to the average of the mastoids off-line. The signal was segmented for each subject into $50 \mathrm{~ms}$ periods starting $100 \mathrm{~ms}$ before stimulus presentation. Each segment was baseline-corrected using the mean voltage during the $100 \mathrm{~ms}$ pre-stimulus period. Segments with an amplitude drift exceeding \pm $100 \mu \mathrm{V}$ at any channel were automatically rejected as were trials on which the base-to-peak EOG amplitude was $>100 \mu \mathrm{V}$. The average number of trials rejected across subjects for each task were as follows: Task $1(2$, $\mathrm{SD}=3.2)$, Task $2(3, \mathrm{SD}=2.4)$, Task $3(15, \mathrm{SD}=2.1)$, Task $4(18, \mathrm{SD}=4.3)$.

For each subject average waveforms to oddballs and frequents for each of the four experimental tasks were calculated ultimately creating 8 ERP waveforms per sub- 
ject (oddball, frequent $\times 4$ tasks). Due to the lack of distinct peaks for some of the subjects ERPs mean amplitudes were calculated for consecutive $50 \mathrm{~ms}$ regions of the waveforms, extending from $0-50$ to $750-800 \mathrm{~ms}$ poststimulus onset. The main analyses were then conducted on the mean amplitudes (negative or positive) in the following latency regions: 120 - $180 \mathrm{~ms}$ (negative: subsequently labeled N100), 150 - $250 \mathrm{~ms}$ (positive: subsequently labeled P200), 200 - $400 \mathrm{~ms}$ (negative: subsequently labeled N200), $300-600 \mathrm{~ms}$ (positive: subsequently labeled P300). These latency regions were chosen because it was in these regions that the maximum mean amplitudes over all the subjects were found. The literature also guided us in choosing these latencies $[62,70]$.

\section{Results}

\subsection{Behavioral Data}

Accuracy and reaction-time measures are summarized for each task in Table 1. Average RTs for each subject ranged from $340 \mathrm{~ms}$ to $550 \mathrm{~ms}$ across tasks. There were no obvious "outliers" so all data was included in the analysis. Analyses of Variance (ANOVAs) were conducted for both accuracy and for reaction times to correctly identified oddballs with Task (1-4) as the independent variable. Participants were on the whole both accurate (mean values for all tasks were over 90\%) and quick to respond suggesting that they found the experiment relatively easy and that they followed the instructions. There were no significant effects of task on either measure despite the fact that participants appeared to be slower to respond to neutral oddballs in a background of emotional frequents (Task 3 and 4, see Table 1).

\subsection{ERPs}

\subsubsection{Task Effects}

We investigated whether an oddball effect (larger P300 amplitudes to oddballs versus frequents i.e. subtractions were calculated for each subject and task) was found in

Table 1. Behavioral performance across the four experimental tasks (means and S.Ds).

\begin{tabular}{lcc}
\hline \multicolumn{1}{c}{ Task Number } & $\begin{array}{c}\text { Accuracy } \\
\text { (\% correctly } \\
\text { identified oddballs) }\end{array}$ & $\begin{array}{c}\text { Reaction Time in ms } \\
\text { (to correctly } \\
\text { identified oddballs) }\end{array}$ \\
\hline 1 (Angry face as oddball) & $92.7(3.9)$ & $462.1(66.2)$ \\
$\begin{array}{l}\text { 2. (Fearful face as oddball) } \\
\begin{array}{l}\text { 3 (Neutral face as oddball, } \\
\text { angry as frequent) }\end{array}\end{array}$ & $91.9(3.5)$ & $463.9(55.4)$ \\
$\begin{array}{l}\text { 4. (Neutral face as oddball, } \\
\text { fearful as frequent) }\end{array}$ & $90.1(4.3)$ & $488.7(62.9)$ \\
\hline
\end{tabular}

Accuracy $=\%$ correctly identified oddballs minus $\%$ mistakes (i.e. false responses to the frequents plus misses to the oddballs). all four experimental tasks. A GLM ANOVA was conducted using 3 independent variables: Task (4 levels), Hemisphere (3 levels: Left, Center, Right) and Site (1 8). A main Task effect $(F(3,652)=20.9, p<0.001)$ emerged and post hoc tests (with Bonferroni correction) confirmed that an oddball effect was only found for Task 1 (where the oddballs were an angry face in a background of neutral frequents) and not for the other 3 tasks ( $\mathrm{p}<$ 0.001 for all comparisons). A main Site effect (F $(5,652)$ $=3.8, \mathrm{p}<0.005)$ suggested that the oddball effect was more frontally distributed (especially at F3, $\mathrm{p}<0.001$ for all site comparisons with bonferroni correction). However, no significant interactions emerged between Hemsiphere or Site and Task.

\subsubsection{Correlations with Questionnaire Subscales and ERP Components}

Rather than putting the participants into specific attachment, autonomy, depression and/or anxious groups we investigated the correlations between the participants ERP components (amplitudes and latencies in Task 1 where the oddball effect was found) and their (continuous) scores on the ASQ-, ACS-30- and SCL-90-subscales. Eugène et al. [49] (among others) have highlighted the need to report individual rather than just group data due to the wide range of individual differences typically found in studies where participants must respond to emotional stimuli. We have followed this suggestion and directly assessed the relationship between participants' scores on various attachment-related questionnaire measures and ERP components rather than placing participants into extreme attachment style groups. Pearson Product Moment correlation matrices were carried out for Task 1 and all subscales (see Tables 2-4). No clear pattern emerged for either the ERP amplitudes to frequent stimuli or for ERP latency measures, so we focus below on ERP amplitudes to oddballs in Task 1.

\subsection{Attachment}

Anxious attachment correlated negatively with N100 (at F3 and F4) and positively with P300 amplitude (at F3 and $\mathrm{Cz}$ ). For Avoidant attachment, no significant effects emerged. For Secure attachment, the opposite pattern to that found for anxious attachment emerged, that is a positive correlation with $\mathrm{N} 100$ amplitude (at F4 and Pz) and a negative correlation with $\mathrm{P} 300$ amplitude (at $\mathrm{Cz}$ and Oz; see Table 2).

\subsection{Partialing Out Anxiety}

When Anxiety (SCL-90) was partialed out the patterns given above (Attachment results) found between N100 amplitude and both secure and anxious attachment disappeared, while those found for P300 amplitude remained 
Table 2. Correlation matrix of ERP component amplitudes to oddballs in Task 1 where the oddball was an angry face in a background of neutral frequents with the scores on the 3 index scores of the ASQ. Also included: correlations when trait anxiety (SCL-90) was partialled out.

\begin{tabular}{|c|c|c|c|}
\hline & Secure & Avoidant & Anxious \\
\hline \multicolumn{4}{|c|}{ Electrode site given when correlation is significant plus the size and direction of the effect } \\
\hline N100 & $\mathrm{Pz}(\mathrm{r}=+0.52)^{* *}, \mathrm{~F} 4(\mathrm{r}=+0.41)^{*}$ & n.s. & $\mathrm{F} 3(\mathrm{r}=-0.48)^{*}, \mathrm{~F} 4(\mathrm{r}=-0.43)^{*}$ \\
\hline P200 & $\mathrm{Fz}(\mathrm{r}=+0.51)^{*}, \mathrm{Oz}(\mathrm{r}=-0.66)^{*}$ & n.s. & n.s. \\
\hline N200 & $\mathrm{F} 3(\mathrm{r}=+0.47)^{*}, \mathrm{Fz}(\mathrm{r}=+0.55)^{*}$ & n.s. & n.s. \\
\hline P300 & $\mathrm{Cz}(\mathrm{r}=-0.41)^{*}, \mathrm{Oz}(\mathrm{r}=-0.72)^{* *}$ & n.s. & $\mathrm{F} 3(\mathrm{r}=+0.49)^{*}, \mathrm{Cz}(\mathrm{r}=+0.42)^{*}$ \\
\hline \multicolumn{4}{|c|}{ And with with trait anxiety (SCL-90) partialled out: } \\
\hline N100 & n.s. & n.s. & n.s. \\
\hline P200 & $\mathrm{Pz}(\mathrm{r}=+0.42)^{*}$ & n.s. & n.s. \\
\hline N200 & n.s. & n.s. & n.s. \\
\hline P300 & $\mathrm{Fz}(\mathrm{r}=-0.50)^{*}$ & $\mathrm{Fz}(+0.51)^{*}$ & $\mathrm{~F} 3(\mathrm{r}=+0.58)^{*}, \mathrm{Fz}(\mathrm{r}=+0.52)^{*}$ \\
\hline
\end{tabular}

${ }^{*} \mathrm{p}<0.05, \stackrel{*}{*} \mathrm{p}<0.01 ;$ n.s. $=$ non-significant.

Table 3. Correlation matrix of ERP component amplitudes to oddballs in Task 1 where the oddball was an angry face in a background of neutral frequents with the scores on the 3 subscales of the ACS-30.

\begin{tabular}{|c|c|c|c|}
\hline & Self Awareness & Sensitivity to Others & Capacity for managing new situations \\
\hline & \multicolumn{3}{|c|}{ Electrode site given when correlation is significant plus the size and direction of the effect } \\
\hline N100 & $\mathrm{Fz}(\mathrm{r}=+0.53)^{*}, \mathrm{Cz}(\mathrm{r}=+0.55)^{* *}$ & n.s. & $\mathrm{C} 3(\mathrm{r}=+0.38)^{*}, \mathrm{Cz}(\mathrm{r}=+0.55)^{* *}$ \\
\hline & $\mathrm{Pz}(\mathrm{r}=+0.43)^{*}$ & & $\mathrm{Pz}(+0.47)^{*} \mathrm{~F} 4(+0.5)^{* *}, \mathrm{C} 4(+0.46)^{* *}$ \\
\hline P200 & n.s. & n.s. & n.s. \\
\hline N200 & n.s. & n.s. & $\mathrm{Fz}(\mathrm{r}=+0.53)^{*}, \mathrm{C} 4(\mathrm{r}=+0.37)^{*}$ \\
\hline P300 & $\mathrm{Cz}(\mathrm{r}=-0.38)^{*}, \mathrm{Pz}(\mathrm{r}=-0.39)^{*}$ & $\mathrm{Oz}(\mathrm{r}=+0.55)^{*}$ & $\mathrm{Cz}(\mathrm{r}=-0.39)^{*}, \mathrm{Oz}(\mathrm{r}=-0.51)^{*}$ \\
\hline
\end{tabular}

${ }^{*} \mathrm{p}<0.05,{ }^{* *} \mathrm{p}<0.01 ;$ n.s. $=$ non-significant.

Table 4. Correlation matrix of ERP component amplitudes to oddballs in Task 1 where the oddball was an angry face in a background of neutral frequents with the depression and anxiety scores from the SCL-90.

\begin{tabular}{lll}
\hline & Depression & Anxiety \\
\hline N100 & Electrode site given when correlation is significant plus the size and direction of the effect \\
\hline P200 & $\mathrm{Oz}(\mathrm{r}=+0.54)^{*}$ & $\mathrm{Pz}(\mathrm{r}=-0.46)^{* *}, \mathrm{C} 4(\mathrm{r}=-0.42)^{*}$ \\
N200 & $\mathrm{Oz}(\mathrm{r}=+0.53)^{*}$ & $\mathrm{Oz}(\mathrm{r}=+0.68)^{* *}$ \\
P300 & $\mathrm{Oz}(\mathrm{r}=+0.68)^{* *}$ & $\mathrm{~F} 3(\mathrm{r}=-0.63)^{* *}, \mathrm{Fz}(\mathrm{r}=-0.67)^{* *}, \mathrm{Oz}(\mathrm{r}=+0.63)^{* *}$ \\
\hline
\end{tabular}

${ }^{*} \mathrm{p}<0.05,{ }^{* *} \mathrm{p}<0.01 ;$ n.s. $=$ non-significant.

(still negatively correlated with secure attachment at F3 and positively correlated with anxious attachment at F3 and Fz; see Table 2).

\subsection{Autonomy-Connectedness}

Self-Awareness and Capacity for managing new situations showed the same pattern with ERP component amplitudes as was found for the Secure ASQ-subscale, namely positive correlations with N100 amplitude (but more widely-spread over the scalp than was the case for the Secure ASQ-subscale: for Task 1 at $\mathrm{Fz}, \mathrm{Cz}$ and $\mathrm{Pz}$ for Self-Awareness, and at F4, C3, Cz, C4, and Pz for Capacity for managing new situations) and negative correlations with $\mathrm{P} 300$ amplitude (at $\mathrm{Cz}, \mathrm{Pz}$, and $\mathrm{Oz}$ for Self-Awareness, and at $\mathrm{Cz}$ and $\mathrm{Oz}$ for Capacity for man- aging new situations; see Table 3).

\subsection{Anxiety and Depression}

Stronger correlations emerged for Anxiety than for Depression (SCL-90). These followed exactly the same pattern as was found for anxious attachment reported above with negative correlations found for N100 amplitude (at $\mathrm{Pz}$ and $\mathrm{C4}$ ) and positive correlations for P300 (at Oz; see Table 4).

\subsection{Correlations between the Subscales}

Inspection of the subscale responses revealed various high correlations, and of main interest here, between Anxiety (SCL-90) and Anxious attachment (ASQ) ( $\mathrm{r}=$ $+0.64, \mathrm{p}<0.001$; see Table 5). 
Table 5. Correlation matrix for the 3 questionnaires.

\begin{tabular}{llll}
\hline & & \multicolumn{1}{c}{ ASQ } & Secure \\
\cline { 2 - 4 } Questionnaire (subscale) & Anxious & Avoidant & \\
ASQ (Attachment Style Questionnaire) & & & $\mathrm{r}=-0.68^{* *}$ \\
Anxious & $\mathrm{r}=1$ & $\mathrm{r}=1$ & $\mathrm{r}=-0.44^{*}$ \\
Avoidant & $\mathrm{r}=+0.48^{*}$ & $\mathrm{r}=-0.44^{*}$ & $\mathrm{r}=1$ \\
Secure & $\mathrm{r}=-0.68^{* *}$ & & $\mathrm{r}=+0.60^{* *}$ \\
ACS-30 (Autonomy-Connectedness scale) & & $\mathrm{r}=-0.44^{*}$ & $\mathrm{r}=-0.49^{*}$ \\
Self awareness & $\mathrm{r}=-0.60^{* *}$ & $\mathrm{n} . \mathrm{s}$. & $\mathrm{r}=+0.69$ \\
Sensitivity to others & $\mathrm{r}=+0.68^{* *}$ & $\mathrm{r}=-0.48^{*}$ & $\mathrm{r}=-0.65^{* *}$ \\
Capacity For Managing New Situations & $\mathrm{r}=-0.70^{* *}$ & & $\mathrm{r}=-0.81^{* *}$ \\
SCL-90 (Symptom Checklist-90) & & $\mathrm{r}=+0.58^{* *}$ & \\
Depression & $\mathrm{r}=+0.72^{* *}$ & $\mathrm{n} . \mathrm{s}$. & \\
Anxiety & $\mathrm{r}=+0.64^{* *}$ & &
\end{tabular}

\section{Discussion}

The current study investigated the relationships between participants' scores on attachment-style and autonomysubscales and their processing emotionally-threatening faces as reflected by their ERPs to angry oddballs (Task 1). We expected that those scoring highly on anxious attachment would exhibit heightened N100/P200 (increased attention) and reduced P300 (reduced elaboration) amplitudes, thus supporting the attentional bias theory for anxiety. We found the opposite pattern, i.e., reduced N100 and heightened P300 amplitudes were related to anxious attachment. Furthermore, we expected that high levels of avoidant attachment would be related to suppression of stimulus processing resulting in a flattening of the overall waveform (i.e. both reduced N100 and P300 amplitudes) but found no siginificant effects with avoidant attachment. Finally, we expected that participants with both high levels of secure attachment and autonomy would show less attention to but more elaborate later processing of task-relevant stimuli, reflected in N100s smaller than those for anxiously attached subjects and larger P300s. We found the opposite pattern, namely larger N100 and smaller P300 amplitudes with secure attachment and autonomy. These findings were evident at selected electrode sites. These results suggest that our hypotheses were, at best, only partially supported. We attempt to interpret these findings below.

\subsection{Interpretation of Findings}

People scoring higher on secure attachment and autonomy scales showed enhanced early attention to the threat (angry face) as reflected in their enhanced N100 amplitudes and subsequent withdrawal or disengagement from that threat (reflected in their reduced P300 amplitudes). We expected these findings for those scoring highly on anxious attachment. The findings for secure attachment coupled with those for anxious attachment (smaller N100 and larger P300s) suggest that our data do not support the attentional bias/cognitive avoidance theory [26,71] typically found in behavioral tasks in people with high trait anxiety, assuming of course that N100 amplitude reflects early attention and P300 amplitude engagement/ disengagement from pertinent stimuli. It is the case that ERP and behavioral/reaction time (RT) data do not always converge (P300 latency tends to correlate with RT chiefly when response accuracy is required, [72]) and indeed we obtained no significant effects for our RT data. Perhaps our tasks were too simplistic to substantially affect RTs in our student participants.

Our results do however support previous findings of larger late positive (P300) amplitudes to threatening stimuli in anxious compared to healthy control groups (see e.g. [50], P200 data). Indeed, in the current study a positive correlation between P300 amplitude and anxious attachment remained even when anxiety was partialed out. This suggests that despite high correlations between anxiety and anxious attachment questionnaire scores the latter might have an independent and/or additive function in how anxious brains process threatening faces. The fact that high scorers on the anxiety subscale (SCL-90) in our study processed the angry faces very similarly to those scoring highly on the anxious attachment subscales, is interesting, and suggests that processes underlying anxious attachment and anxiety may overlap.

Also other authors reported reduced attention to threatening stimuli in those with anxious attachment. Main et al. [20] found that anxiously as well as avoidantly attached children looked away from attachment-related photographs. Kirsch and Cassidy [73] found the same avoidance of attachment-related information in their insecurely attached child participants, while in their fMRI study Gillath et al. [74] found reduced activation in orbitofrontal cortex in their anxiously-attached participants.

As suggested in the Introduction, the literature does not always support the attentional bias theory of (general) anxiety or the approach/avoidance to threat expected of anxious/avoidantly-attached individuals $[20,25]$. Threat 
approach or avoidance seems to depend largely on the con-text, the specific task demands and the length of stimulus exposure time.

That high secure attachment was associated with quick attention to the angry faces but not with subsequent dwelling on them, suggests that secure attachment coincides with either high awareness of the environment and/ or of oneself (as is also indicated by high self-awareness, one of the ACS-subscales). This may then facilitate the choice of the optimal reaction: avoiding angry people as a good strategy for avoiding confrontation in relationships, an adaptive strategy. The fact that higher levels of anxious attachment were related to lack of attention to the angry faces initially, but dwelling on them later suggests that anxious attachment implies not picking up the cues in the environment that someone is displeased with you. This could have maladaptive consequences. Most people given the chance would go out of their way to avoid angry people. Also, larger P300 amplitudes for those scoring highly on anxious attachment subscales may suggest that the angry oddballs are very relevant to these participants, perhaps more relevant than is the case for those scoring highly on the secure subscales. An alternative explanation could be that in the anxiously-attached positive shifts in the ERP occur very early after stimulus presentation resulting in reduced N100s and continue to late in the epoch resulting in larger P300s (this interprettation agrees with that [58]).

\subsection{Links with the Attachment Style and ERP Literature}

Cassidy [75,76] has suggested that attachment theory offers a theoretical framework for how Generalized Anxiety Disorder can develop in adulthood. It also appears to be the case that the attachment system is activated most in times of stress or threat and it is in these circumstances that the fear system is also activated [17]. In the current study it seems that attention to potentially threatening material is reduced in participants who score highly on anxious attachment subscales in general. This has also been found by other researchers using different experimental designs (e.g. [20,73,74]).

We found significant correlations at frontal, central and parietal/occipital sites. These scalp sites have been located to sources based in medial frontal cortex and temporal lobe regions both of which have in turn been linked with aspects of processing (especially emotional) stimuli but also aspects of (especially autobiographical and/or episodic) memory. Coan [77] has suggested that the parent acts as a "surrogate prefrontal cortex" in the child's early life, before the frontal cortex has fully developed. If secure attachment has not been achieved in these early years Coan seems to suggest that the frontal cortex will never work properly, a sobering thought. Zhang et al. [57] also found that ERP amplitudes differed at frontal sites according to participants' attachment-orientation. These findings, including ours, combine to suggest that the (pre) frontal cortex and the amygdala may be the crucial neurological sites involved in attachment and that threatprocessing pathways in the brain may overlap or map on to these attachment regions in some way. However, we need to be cautious in our interpretations based on only a few electrode sites.

A much broader network including the thalamus, hippocampus, locus coeruleus, periaqueductal gray and more may be involved in aspects of attention, startle, escape and avoidance [78,79] all of which may be very relevant to the attachment system and how it deals with external (and also perhaps internal) threat. The ERP has the disadvantage in that it tells us relatively little about which areas in the brain are involved in tasks assessing emotional-processing. Perhaps a combination of brainimaging techniques could be used in future studies while at the same time we must recognize that our current techniques still do not allow us to examine the amygdala (and other brain regions) in a fine-grained way [32].

\subsection{Comparison with Other ERP/Attachment Style Studies}

Zilber et al. [58] found heightened LPP amplitudes to negative pictures in anxiously-attached participants and our data, despite the fact that we used completely different methodology, stimuli etc, support these authors' findings in that we too found a heightened late positivity, (P300 to angry faces) in participants scoring highly on both anxious attachment and trait anxiety subscales. This positive correlation between P300 amplitude and anxious attachment remained even after trait anxiety was partialed out.

Zhang et al.'s [57] study was closer than Zilber et al.'s to ours in that they also used emotional adult facial expressions. Our tasks are not however directly comparable to Zhang et al.'s in that they used a backward masking task (compared to our oddball task), divided their subjects into groups using an entirely different questionnaire to the one we used to assess attachment (namely the ECR, [24]), and used different stimuli and presentation rates (ours were longer at $200 \mathrm{~ms}$ compared to both their supraliminal $170 \mathrm{~ms}$ SOA and subliminal $34 \mathrm{~ms}$ SOA presentations). All these factors can have a major impact on whether the attentional bias to threat will be observed or not). Therefore replication of both studies is desirable.

Fraedrich et al. [62] found larger P300 amplitudes to infant faces compared to flower stimuli in secure (versus insecure) mothers, but no differences between positive and negative expressions on those face stimuli. In contrast to Fraedrich et al. we found smaller P300s with secure attachment in student participants to angry adult faces as 
oddballs. Fraedrich et al. also measured the face-specific N170 component and found it to be heightened in their insecure (versus secure) mothers while the attention-related N100 was smaller in the insecure group. Fraedrich et al.'s study and ours differed from each other on many levels including the most obvious, that they used mothers as participants and infant faces (positive, negative, neutral expressions) as stimuli. They also classified their participants as insecure or securely attached by means of the Adult Attachment Projective (AAP; [80]), a projecttive rather than a questionnaire measure. Furthermore, a three-stimulus (as opposed to our two-stimulus) oddball task was also employed. Finally, we did not measure the face-specific N170 component, something we will consider doing in future experiments.

\subsection{Limitations and Future Research}

Orozco and Ehlers [41] stated that the amplitudes and latencies of the ERP components depend on the type of emotion depicted by the stimuli, the gender of those stimuli and of the participant tested. We chose female stimuli and only three emotional expressions (angry, fearful, and neutral) in an attempt to simplify the task yet make it relevant enough for participants with different levels of attachment styles. Future research should replicate the findings depicted here while including an all neutral baseline task and a wider number of scalp electrodes to better investigate activity and enable source analyses to be carried out. It might also be interesting to use a multimethod approach for measuring attachment, i.e., aside from self-report measures (this study) one might use the Adult Attachment Interview [81].

The Oddball Effect, i.e. larger P300 amplitudes to oddballs than to frequents was only found to be signifycant for Task 1 when the oddballs were angry faces in a background of neutral. This could mean that both novelty (oddball) and emotion (anger) enhanced the P300, a finding supported by a substantial literature (i.e. larger P300s are typically found to oddballs and emotionally-toned stimuli versus frequents and neutrals - see for example [34, $35,40]$ ). The findings could also mean that there were insufficient trials (see Methods) per task to obtain the oddball effect in all four tasks, and indeed there were fewer trials available due to artifacts in Task 3 and 4. Future experiments should use more trials per task in order to enhance both the signal-to-noise ratio and the chance of obtaining more clear-cut early ERP components.

We chose static female faces for a number of reasons not least because the first attachment figure is typically the mother. We expect that the relatively clear patterns found with a group of 25 participants would appear even more clearly with a larger sample. Stronger findings have also been found for moving as opposed to static faces in the literature [32].

\section{Conclusions}

In conclusion, this study, despite its limitations has shown that there are different patterns of emotional processing related to anxious, avoidant and secure attachment. The exact mechanisms behind these findings are unknown but future research is clearly important to tease out the associations between attachment dimensions and attention to and further processing of emotionally-relevant stimuli. The ERP is ideal for this because it can highlight what happens before a response is required. The strongest effects were seen here for angry oddballs and we would like to suggest that this emotion might be the most fruitful for future work on the differences between information processing as assessed by ERPs and attachment style but that other emotional, perhaps personally-relevant and/or attachment-related stimuli may also be useful and should not be discarded. Researchers are becoming more aware of how essential it is to investigate individual differences in information processing. The current study shows that this is indeed the case and that more focus on individual differences between participants (rather than group effects) should be encouraged in future ERP research.

In summary, we found that anxiety clearly influenced attentional processes (both anxiously attached and trait anxious participants had reduced attention to threatening, angry oddballs as reflected by their reduced N100 amplitudes). Indeed trait anxiety seemed to be most important for this effect because the N100 amplitude effects for anxious attachment disappeared when trait anxiety was partialed out. P300 amplitude effects remained even after trait anxiety was partialed out with larger P300s to angry oddballs in those scoring highly on anxious attachment. We suggested that those who are anxiously attached might have limited early attention to threat but may dwell on it later and indeed that such stimuli might be very relevant to them. Secure attachment and autonomy correlated with heightened attention to threat (larger N100s) and smaller P300s. We suggested that secure, autonomous individuals are highly tuned in to threat in their environments but that they do not dwell/focus on it; and that this might be very adaptive: given the choice most people would want to avoid threat.

Clearly, the mechanism underlying trait anxiety, attachment and autonomy are complex and much more fundamental work is required before we can come to any definite conclusions. The current study and a handful of others are beginning to tease out how the attachment and anxiety systems might work. The mechanisms behind how attachment styles develop in the infant and how they persist into adulthood is a field ripe for scientific research The question is: are the brains of individuals with different attachment styles fundamentally different from each other? Time and future research will tell! 


\section{REFERENCES}

[1] J. Bowlby, "Attachment and Loss (Vol. I). Attachment," Hoghart Press, London, 1969.

[2] J. Bowlby, "Attachment and Loss (Vol. II). Separation, Anxiety and Anger," Basic Books, New York, 1973.

[3] J. Bowlby, "Attachment and Loss (Vol. III). Loss, Sadness and Depression," Basic Books, New York, 1980.

[4] J. Bowlby, "Attachment and Loss (Vol. I). Attachment," Basic Books, New York, 1982.

[5] A. T. Beck, "Cognitive Therapy and the Emotional Disorders," International Universities Press, New York, 1976.

[6] Y. Minagawa-Kawai, S. Matsuoka, I. Dan, N. Naoi, K. Nakamura and S. Kojima, "Prefrontal Activation Associated with Social Attachment: Facial-Emotion Recognition in Mothers and Infants," Cerebral Cortex, Vol. 19, No. 2, 2009, pp. 284-292. doi:10.1093/cercor/bhn081

[7] M. W. Baldwin, J. P. R. Keelan, B. Fehr, V. Enns and E. Koh-Rangarajoo, "Social Cognitive Conceptualization of Attachment Working Models: Availability and Accessibility Effects," Journal of Personality and Social Psychology, Vol. ,71 No. 1, 1996, pp. 94-104. doi:10.1037/0022-3514.71.1.94

[8] M. W. Baldwin and B. Fehr, "On the Instability of Attachment Style Ratings," Personal Relationships, Vol. 2, No. 3, 1995, pp. 247-261. doi:10.1111/j.1475-6811.1995.tb00090.x

[9] R. C. Fraley, "A Connectionist Approach to the Organization and Continuity of Working Models of Attachment," Journal of Personality, Vol. 75, No. 6, 2007, pp. 11571180. doi:10.1111/j.1467-6494.2007.00471.x

[10] R. C. Fraley, N. G. Waller and K. A. Brennan, "An Item Response Theory Analysis of Self-Report Measures of Adult Attachment," Journal of Personality and Social Psychology, Vol. 78, No. 2, 2000, pp. 350-365. doi:10.1037/0022-3514.78.2.350

[11] M. D. S. Ainsworth, M. C. Blehar, E. Waters and S. Wall "Patterns of Attachment: A Psychological Study of the Strange Situation," Routledge, New York, 1978.

[12] A. Ward, R. Ramsay and J. Treasure, "Attachment Research in Eating Disorders," British Journal of Medical Psychology, Vol. 73, No. 1, 2000, pp. 35-51. doi:10.1348/000711200160282

[13] A. Bifulco, P. Moran, C. Ball and O. Barnazzani, "Adult Attachment Style: Its Relationship to Clinical Depression," Social Psychiatry and Psychiatric Epidemiology, Vol. 37, No. 2, 2002, pp. 50-59. doi:10.1007/s127-002-8215-0

[14] S. Blatt and K. Levy, "Attachment Theory, Psychoanalysis, Personality Development and Psychopathology," Psychoanalytic Inquiry, Vol. 23, No. 1, 2003, pp. 102-150. doi:10.1080/07351692309349028

[15] M. H. J. Bekker and M. A. L. M. van Assen, "A Short Form of the Autonomy Scale: Properties of the Autonomy-Connectedness Scale (ACS-30)," Journal of Personality Assessment, Vol. 86, No. 1, 2006, pp. 51-60. doi:10.1207/s15327752jpa8601 07

[16] M. H. J. Bekker, N. Bachrach and M. A. Croon, "The Relationship of Antisocial Behaviour with Attachment Styles,
Autonomy-Connectedness and Alexythimia," Journal of Clinical Psychology, Vol. 63, No. 6, 2007, pp. 507-527. doi: $10.1002 /$ jclp.20363

[17] J. Cassidy, J. Lichtenstein-Phelps, N. J. Sibrava, C. L. Thomas Jr. and T. D. Borkovec, "Generalized Anxiety Disorder: Connections with Self-Reported Attachment," Behavioral Therapy, Vol. 40, No. 1, 2009, pp. 3-38.

[18] S. Guttmann-Steinmetz and J. A. Crowell, "Attachment and Externalizing Disorders: A Developmental Psychopathology Perspective," Journal of the American Academy of Child and Adolescent Psychiatry, Vol. 45, No. 4, 2006, pp. 440-450. doi:10.1097/01.chi.0000196422.42599.63

[19] R. Kobak, J. Cassidy, K. Lyons-Roth and Y. Ziv, "Attachment, Stress, and Psychopathology: A Developmental Pathways Model," In: D. Cicchetti and D. J. Cohen, Eds., Developmental Psychopathology: Theory and Method, 2nd Edition, John Wiley \& Sons, Hoboken, 2006, pp. 333-369.

[20] M. Main, N. Kaplan and J. Cassidy, "Security in Infancy, Childhood and Adulthood: A Move to the Level of Representation," In: I. Bretherton and E. Waters, Eds., Growing Points in Attachment Theory and Research Monographs of the Society for Research in Child Development 50 (1-2, Serial No. 209), University of Chicago Press, Chicago, 1985, pp. 66-104.

[21] M. H. J. Bekker and U. Belt, "The Role of AutonomyConnectedness in Anxiety and Depression," Depression and Anxiety, Vol. 23, No. 5, 2006, pp. 274-280. doi: $10.1002 /$ da. 20178

[22] M. H. J. Bekker and M. A. Croon, "The Roles of Autonomy-Connectedness and Attachment Styles in Depression and Anxiety," Journal of Personal and Social Relations, Vol. 27, No. 7, 2010, pp. 908-923. doi: $10.1177 / 0265407510377217$

[23] M. Main, "Cross-Cultural Studies of Attachment Organization: Recent Studies, Changing Methodologies and the Concept of Conditional Strategies," Human Development, Vol. 33, 1990, pp. 48-61. doi:10.1159/000276502

[24] K. A. Brennan, C. L. Clark and P. R. Shaver, "Self-Report Measurement of Adult Romantic Attachment: An Integrative Overview," In: J. A. Simpson and W. S. Rholes, Eds., Attachment Theory and Close Relationships, Guilford Press, New York,1998, pp. 46-76.

[25] M. Dewitte, E. H. Koster, J. De Houwer and A. Buysse, "Attentive Processing of Threat and Adult Attachment: A Dot-Probe Study," Behaviour Research and Therapy, Vol. 45, No. 6, 2007, pp. 1307-1317. doi:10.1016/j.brat.2006.11.004

[26] K. Mogg, P. Philippot and B. P. Bradley, "Selective Attention to Angry Faces in Clinical Social Phobia," Journal of Abnormal Psychology, Vol. 113, No. 1, 2004, pp. 160-165. doi:10.1037/0021-843X.113.1.160

[27] M. Mikulincer, G. Birnbaum, D. Woddis and O. Nachmias, "Stress and Accessibility of Proximity-Related Thoughts: Exploring the Normative and Intraindividual Components of Attachment Theory," Journal of Personality and Social Psychology, Vol. 78, No. 3, 2000, pp. 509-523. doi:10.1037/0022-3514.78.3.509 
[28] A. Besser and B. Priel, "A Multisource Approach to SelfCritical Vulnerability to Depression: The Moderating Role of Attachment," Journal of Personality, Vol. 71, No. 4, 2003, pp. 515-556. doi:10.1111/1467-6494.7104002

[29] K. B. Carnelley, P. R. Pietromonaco and K. Jaffe, "Depression, Working Models of Others and Relationship Functioning," Journal of Personality and Social Psychology, Vol. 66, No. 1, 1994, pp. 127-140. doi:10.1037/0022-3514.66.1.127

[30] J. E. Roberts, I. H. Gotlib and J. D. Kassel, "Adult Attachment Security and Symptoms of Depression: The Mediating Roles of Dysfunctional Attitudes and Low SelfEsteem," Journal of Personality and Social Psychology, Vol. 70, No. 2, 1996, pp. 310-320.

[31] J. Holmes, "Attachment Theory: A Biological Basis for Psychotherapy?” British Journal of Psychiatry, Vol. 163, No. 4, 1993, pp. 430-438. doi:10.1192/bjp.163.4.430

[32] K. N. Ochsner, "Current Directions in Social Cognitive Neuroscience," Current Opinion in Neurobiology, Vol. 14, No. 2, 2004, pp. 254-258. doi:10.1016/j.conb.2004.03.011

[33] J. K. Olofsson, S. Nordin, H. Sequeira and J. Polich, “Affective Picture Processing: An Integrative Review of ERP Findings," Biological Psychology, Vol. 77, No. 3, 2008, pp. 247-265. doi:10.1016/j.biopsycho.2007.11.006

[34] R. Näätänen and T. W. Picton, "N2 and Automatic versus Controlled Processes," In: W. C. McCallum, R. Zappoli and F. Denoth, Eds., Cerebral Psychophysiology: Studies in Event-Related Potentials, Elsevier, New York, 1986, pp. 169-186.

[35] E. Donchin and M. G. H. Coles, "Is the P300 Component a Manifestation of Context Updating?" The Behavioral and Brain Sciences, Vol. 11, No. 3, 1988, pp. 355-425. doi:10.1017/S0140525X00058027

[36] R. Verleger, "On the Utility of P3 Latency as an Index of Mental Chronometry," Psychophysiology, Vol. 34, 1997, pp. 131-156. doi:10.1111/j.1469-8986.1997.tb02125.x

[37] A. Gasbarri, B. Arnone, A. Pompili, A. Marchetti, F. Pacitti, S. S. Calil, C. Pacitti, M. C. Tavares and C. Tomaz, "Sex-Related Lateralized Effect of Emotional Content on Declarative Memory: An Event Related Potential Study," Behavioural Brain Research, Vol. 168, No. 2, 2006, pp. 177-184. doi:10.1016/j.bbr.2005.07.034

[38] M. Falkenstein, J. Hohnsbein and J. Hoormann, "Effects of Choice Complexity on Different Subcomponents of the Late Positive Complex of the Event-Related Potential," Electroencephalography and Clinical Neurophysiology, Vol. 92, No. 2, 1994, pp. 148-160. doi:10.1016/0168-5597(94)90055-8

[39] J. T. Cacioppo, S. L. Crites Jr., W. L. Gardner and G. G. Bernston, "Bioelectrical Echoes from Evaluative Categorizations: I. A Late Positive Brain Potential That Varies as a Function of Trait Negativity and Extremity," Journal of Personality and Social Psychology, Vol. 67, No. 1, 1994, pp. 15-125.

[40] B. N. Cuthbert, H. T. Schupp, M. M. Bradley, N. Birbaumer and P. J. Lang, "Brain Potentials in Affective Picture Processing: Covariation with Autonomic Arousal and Affective Report," Biological Psychology, Vol. 52, 2000, pp.
95-111. doi:10.1016/S0301-0511(99)00044-7

[41] S. Orozco and C. L. Ehlers, "Gender Differences in Electrophysiological Responses to Facial Stimuli," Biological Psychiatry, Vol. 44, No. 4, 1998, pp. 281-289. doi:10.1016/S0006-3223(97)00487-3

[42] D. Palomba, A. Angrilli and A. Mini, "Visual Evoked Potentials, Heart Rate Responses and Memory to Emotional Pictorial Stimuli," International Journal of Psychophysiology, Vol. 27, No. 1, 1997, pp. 55-67. doi:10.1016/S0167-8760(97)00751-4

[43] J. T. Cacioppo, W. L. Gardner and G. G. Bernston, "The Affect System Has Parallel and Integrative Processing Components: Form Follows Function," Journal of Personality and Social Psychology, Vol. 76, No. 5, 1999, pp. 839-855.

[44] M. Soltani and R. T. Knight, "Neural Origins of the P300," Critical Review of Neurobiology, Vol. 14, No. 3-4, 2000, pp. 199-224.

[45] H. Barbas, "Connections Underlying the Synthesis of Cognition, Memory, and Emotion in Primate Prefrontal Cortices," Brain Research Bulletin, Vol. 52, No. 5, 2000, pp. 319-330.

[46] A. R. Damasio, T. J. Grabowski, A. Bechara, H. Damasio, L. L. B. Ponto, J. Parvizi and R. D. Hichwa, "Subcortical and Cortical Brain Activity during the Feeling of SelfGenerated Emotions," Nature Neuroscience, Vol. 3, No. 10, 2000, pp. 1049-1056. doi:10.1038/79871

[47] M. Beauregard, S. Karama, J.-M. Leroux, A. R. Lecours, G. Beaudoin and P. Bourgouin "The Functional Neuroanatomy of Amusement, Disgust, and Sexual Arousal," NeuroImage, Vol. 7, No. 4, 1998, p. S909.

[48] R. D. Lane, E. M. Reiman, G. L. Ahern, G. E. Schwartz and R. J. Davidson, "Neuroanatomical Correlates of Happiness, Sadness, and Disgust," American Journal of Psychiatry, Vol. 154, No. 7, 1997, pp. 926-933.

[49] F. Eugène, J. Lévesque, B. Mensour, J. M. Leroux, G. Beaudoin, P. Bourgouin and M. Beauregard, "The Impact of Individual Differences on the Neural Circuitry Underlying Sadness," NeuroImage, Vol. 19, No. 2 Pt 1, 2003, pp. 354-364.

[50] Y. Bar-Haim, D. Lamy and S. Glickman, "Attentional Bias in Anxiety: A Behavioral and ERP Study," Brain and Cognition, Vol. 59, No. 1, 2005, pp. 11-22. doi:10.1016/j.bandc.2005.03.005

[51] E. Fox, "Processing Emotional Facial Expressions: The Role of Anxiety and Awareness," Cognitive, Affective, and Behavioral Neuroscience, Vol. 2, No. 1, 2002, pp. 52-63. doi:10.3758/CABN.2.1.52

[52] M. Eimer and A. Holmes, "Event-Related Potential Correlates of Emotional Face Processing," Neuropsychologia, Vol. 45, No. 1, 2007, pp. 15-31. doi:10.1016/j.neuropsychologia.2006.04.022

[53] M. Sugiura, R. Kawashima, M. Nakagawa, K. Okada, T. Sato, R. Goto, K. Sato, S. Ono, T. Schormann, K. Zilles and H. Fukuda, "Correlation between Human Personality and Neural Activity in Cerebral Cortex," NeuroImage, Vol. 11, No. 5, 2000, pp. 541-546. doi:10.1006/nimg.2000.0564 
[54] T. Canli, Z. Zhao, J. E. Desmond, E. Kang, J. Gross and J. D. E. Gabrieli, "An fMRI Study of Personality Influences on Brain Reactivity to Emotional Stimuli," Behavioral Neuroscience, Vol. 115, No. 1, 2001, pp. 33-42. doi:10.1037/0735-7044.115.1.33

[55] M. A. L. M. van Assen and M. H. J. Bekker, "Sex Differences in Autonomy-Connectedness: The Role of Personality Factors," Personality and Individual Differences, Vol. 47, No. 1, 2009, pp. 12-17. doi:10.1016/j.paid.2009.01.039

[56] R. E. Mark, "Worry, Information Processing and EventRelated Potentials," Unpublished Ph.D. Thesis, Queens University Belfast, Belfast, 1993.

[57] X. Zhang, T. Li and X. Zhou, "Brain Responses to Facial Expressions by Adults with Different Attachment-Orientations," NeuroReport, Vol. 19, No. 4, 2008, pp. 437-441. doi:10.1097/WNR.0b013e3282f55728

[58] A. Zilber, A. Goldstein and M. Mikulincer, "Adult Attachment Orientations and the Processing of Emotional Pictures-ERP Correlates," Personality and Individual Differences, Vol. 43, No. 7, 2007, pp. 1898-1907. doi:10.1016/j.paid.2007.06.015

[59] P. J. Lang, M. M. Bradley and B. N. Cuthbert, "International Affective Picture System (IAPS): Instruction Manual and Affective Ratings. Technical Report A-4," The Center for Research in Psychophysiology, University of Florida, Gainesville, 1999.

[60] V. Zayas, Y. Shoda, W. Mischel, L. Osterhout and M. Takahashi, "Neural Responses to Partner Rejection Cues," Psychological Science, Vol. 20, No. 7, 2009, pp. 813-821. doi:10.1111/j.1467-9280.2009.02373.x

[61] M. Kutas and S. A. Hillyard, "Reading Senseless Sentences: Brain Potentials Reflect Semantic Incongruity," Science, Vol. 207, No. 4427, 1980, pp. 203-205. doi:10.1126/science.7350657

[62] E. M. Fraedrich, K. Lakatos and G. Spangler, "Brain Activity during Emotion Perception: The Role of Attachment Representation," Attachment and Human Development, Vol. 12, No. 3, 2010, pp. 231-48. doi:10.1080/14616731003759724

[63] J. A. Feeney, P. Noller and M. Hanrahan, "Assessing Adult Attachment," In M. B. Sperling and W. H. Berman, Eds., Attachment in Adults, Guilford Press, New York, 1994, pp. 128-152.

[64] A. Fossati, J. A. Feeney, D. Donati, M. Donini, L. Novella, M. Bagnato, I. Carretta, B. Leonardi, S. Mirabelli and C. Maffei, "Personality Disorders and Adult Attachment Domains in a Mixed Psychiatric Sample: A Multivariate Study," Journal of Nervous and Mental Disease, Vol. 191, No. 1, 2003, pp. 30-37. doi:10.1097/00005053-200301000-00006

[65] M. H. J. Bekker, J. J. Willemse and J. W. De Goeij, "The Role of Individual Differences in Particular AutonomyConnectedness in Women's and Men's Work-Family Balance," Women Health, Vol. 50, No. 3, 2010, pp. 241-261. doi:10.1080/03630242.2010.480902

[66] M. H. J. Bekker and M. A. L. M. van Assen, "AutonomyConnectedness and Gender," Sex Roles, Vol. 59, No. 7-8, 2008, pp. 532-544. doi:10.1007/s11199-008-9447-x
[67] L. R. Derogatis, "SCL-90 Administration, Scoring and Procedures Manual-I," Johns Hopkins, Baltimore, 1977.

[68] D. Matsumoto and P. Ekman, "Japanese and Caucasian Facial Expressions of Emotion and Neutral Faces (JACFEE and JACNeuF)," 1988. http://www.paulekman.com

[69] H. A. Jasper, "The Ten-Twenty Electrode System of the International Federation," Electroencepholography and Clinical Neurophysiology, Vol. 10, No. 2, 1958, pp. 371375.

[70] J. K. Olofsson, S. Nordin, H. Sequeira and J. Polich, “Affective Picture Processing: An Integrative Review of ERP Findings," Biological Psychology, Vol. 77, No. 3, 2008, pp. 247-265. doi:10.1016/j.biopsycho.2007.11.006

[71] J. M. G. Williams, F. N. Watts, C MacLeod and A. Mathews, "Cognitive Psychology and Emotional Disorders," Wiley, Chichester, 1988.

[72] S. A. Hillyard and T. W. Picton, "Electrophysiology of Cognition," In: S. R. Geiger, Ed., Handbook of Physiology: A Critical, Comprehensive Presentation of Physiological Knowledge and Concepts, American Physiology Association, New York, 1987, pp. 519-584.

[73] S. J. Kirsh and J. Cassidy, "Preschoolers' Attention to and Memory for Attachment-Relevant Information," Child Development, Vol. 68, No. 6, 1997, pp. 1143-1153. doi: $10.2307 / 1132297$

[74] O. Gillath, S. A. Bunge, P. R. Shaver, C. Wendelken and M. Mikulincer, "Attachment-Style Differences in the Ability to Suppress Negative Thoughts: Exploring the Neura Correlates," NeuroImage, Vol. 28, 2005, pp. 835-847.

[75] J. Cassidy, "Attachment and Generalized Anxiety Disorder," In: D. Cicchetti and S. Toth, Eds., Rochester Symposium on Developmental Psychopathology: Emotion, Cognition, and Representation, University of Rochester Press, Rochester, 1995, pp. 343-370.

[76] J. Cassidy and P. R. Shaver, "Handbook of Attachment: Theory, Research, and Clinical Applications," Guilford Press, New York, 1999.

[77] J. A. Coan, "Toward a Neuroscience of Attachment," In: J. Cassidy and P. R. Shaver, Eds., Handbook of Attachment: Theory, Research, and Clinical Applications, 2nd Edition, Guilford Press, New York, 2008, pp. 241-265.

[78] M. Davis and P. J. Whalen, "The Amygdala: Vigilance and Emotion," Molecular Psychiatry, Vol. 6, No. 1, 2001, pp. 13-34. doi:10.1038/sj.mp.4000812

[79] N. McNaughton and P. J. Corr, "A Two-Dimensional Neuropsychology of Defense: Fear/Anxiety and Defensive Distance," Neuroscience and Biobehavioral Reviews, Vol. 28, No. 3, 2004, pp. 285-305.

doi:10.1016/j.neubiorev.2004.03.005

[80] C. George and M. West, "The Development and Preliminary Validation of a New Measure of Adult Attachment: The Adult Attachment Projective," Attachment and Human Development, Vol. 3, No. 1, 2001, pp. 30-61. doi:10.1080/14616730010024771

[81] C. George, N. Kaplan and M. Main, "An Adult Attachment Interview,” University of California, Berkeley, 1984. 